\title{
Marine microplastics bound dioxin-like chemicals: Model explanation and risk assessment
}

\author{
Qiqing Chen ${ }^{\mathrm{a}, \mathrm{b}}$, Haibo Zhang ${ }^{\mathrm{c}, *}$, Annika Allgeier ${ }^{\mathrm{b}}$, Qian Zhou ${ }^{\mathrm{d}}$, Jacob D. Ouellet ${ }^{\mathrm{b}}$, \\ Sarah E. Crawford ${ }^{\mathrm{b}}$, Yongming Luo ${ }^{\mathrm{e}}$, Yi Yang ${ }^{\mathrm{a}}$, Huahong Shi ${ }^{\mathrm{a}}$, Henner Hollert ${ }^{\mathrm{b}, *}$ \\ a State Key Laboratory of Estuarine and Coastal Research, East China Normal University, Shanghai 200062, China \\ ${ }^{\mathbf{b}}$ Department of Ecosystem Analysis, Institute for Environmental Research, ABBt - Aachen Biology and Biotechnology, RWTH Aachen University, 1 Worringerweg, 52074 \\ Aachen, Germany \\ ${ }^{\mathrm{c}}$ Key Laboratory of Soil Contamination Bioremediation of Zhejiang Province, School of Environmental \& Resource Sciences, Zhejiang Agriculture and Forestry University, \\ Hangzhou 311300, China \\ ${ }^{\mathrm{d}}$ Key Laboratory of Coastal Environmental Processes and Ecological Remediation, Yantai Institute of Coastal Zone Research, Chinese Academy of Sciences, Yantai \\ 264003, China \\ ${ }^{\mathrm{e}}$ Nanjing Institute of Soil Science, Chinese Academy of Sciences, Nanjing 210008, China
}

\section{G R A P H I C A L A B S T R A C T}

Dioxin-like chemical concentrations increasing

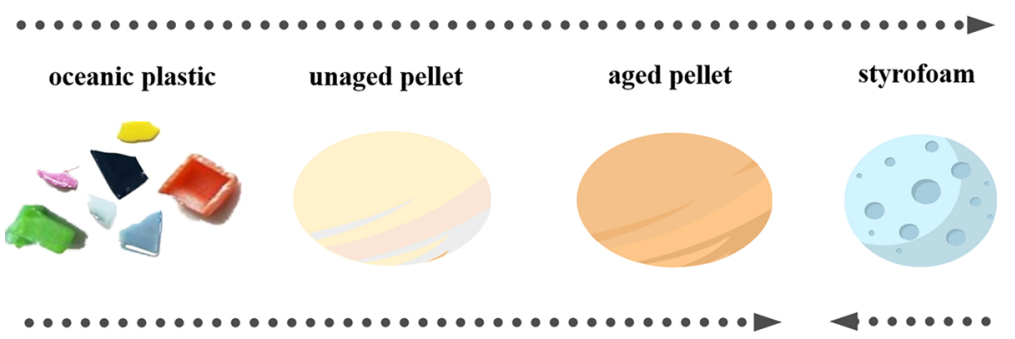

Dioxin-like effect increasing

DLC leaching $>$ EC10

\section{A R T I C L E I N F O}

\section{Keywords:}

Microplastics

Dioxin-like chemicals

Styrofoam

Aging effect

\begin{abstract}
A B S T R A C T
Microplastics have become one of the most pervasive emerging pollutants in the marine environment because of their wide occurrence and high sorption ability for hydrophobic organic contaminants (HOCs). Among the associated HOCs, dioxin-like chemicals (DLCs) can pose severe health risks; however, information on effects of microplastics bound DLCs is lacking. To fill this knowledge gap, this study integrated chemical analysis and in vitro bioassays to elucidate the potential dioxin-like effects of microplastics bound DLCs. Chemical analysis results demonstrated that styrofoams possessed significantly greater DLCs than other coastal or open ocean plastic particles. This was probably due to the presence of additives and greater sorption ability of expanded polystyrene. However, styrofoams did not show as strong dioxin-like effects as predicted by the bioanalysis equivalent model in bioassays. This could be attributed to the decreased DLC bioavailability and increased competition with the presence of styrene oligomers. Besides, bioassay results also demonstrated that aging increased the associated DLC concentrations, since extra sorption from surrounding environment occurred during prolonged retention periods. Finally, it was estimated that the leaching of DLCs could induce dioxin-like effects in marine organisms under $100 \%(11 / 11)$ and $18 \%(2 / 11)$ scenarios for aged pellets and styrofoams through aqueous or dietary exposures.
\end{abstract}

\footnotetext{
* Corresponding authors.

E-mail addresses: hbzhang@zafu.edu.cn (H. Zhang), henner.hollert@bio5.rwth-aachen.de (H. Hollert).
} 


\section{Introduction}

The widespread occurrence of plastic debris in the environment has raised concern about their potential for causing serious environmental and health hazard [1]. Plastic debris can be separated into microplastics (0.1-5 mm), mesoplastics (5-25 mm), and macroplastics ( $>25 \mathrm{~mm}$ ) $[2,3]$. Moreover, when microplastics can further fragment or degrade to even smaller nanoplastics $(<100 \mathrm{~nm})[4,5]$. Since the pioneering study on microplastics in seas published by Richard Thompson in 2004 [6], the severity of microplastic contamination in the oceans and coastal regions has been widely demonstrated [7]. Plastic debris is widely dispersed in the marine environment. Microplastics are stranded or buried on beaches or found floating on open ocean surfaces [8,9]. Different marine ambient conditions can alter the shape, surface area, and coloration of the microplastic particles. Moreover, the sorbed pollutants and additive contents in microplastics can also be affected and subsequently lead to different environmental impact and toxicological consequences [10-12]. For example, enhanced neurotoxicity and behavioral abnormality, increased pollutant metabolites concentrations, and endocrine disrupting effects have already been observed to occur in zebrafish (Danio rerio) [13,14], goby fish (Pomatoschistus microps) [15], and medaka (Oryzias latipes) [16], respectively.

Due to the high sorption capacity of plastics toward hydrophobic organic contaminants (HOCs), microplastics act as a "vector" that are loaded with numerous organic pollutants $[17,18]$. Polymeric particles contain additives used to improve their performance, and the release of these organic additives may take place during the life of the plastics or after their disposal [19]. Plasticizers, flame retardants, and antimicrobial agents are commonly found toxic additives [20]. In addition to the release of organic additives, microplastic polymers can also behave like passive samplers, accumulating "environmental pollutants" present in the surrounding compartments [21,22]. These environmental potentially toxic substances (PoTSs) could further exacerbate the complications in all stages of marine plastic debris [12]. With their relatively longer half-life than most natural substances or products [23], microplastics can also enhance the transport of associated chemicals by "sorb and shuttle" processes [24]. These processes, whereby contaminants adsorb to microplastics and are transported, can result in the extensive distribution of chemical-associated microplastic, even to remote regions like the Arctic [25] and to every level of food chain in the marine environment [7]. Upon ingestion, the plastic-associated organic compounds can become bioavailable to organisms and can accumulate in the food chain $[13,14,26]$.

There is increasing number of studies reporting HOCs on microplastics in recent years. Among these HOCs, polyaromatic hydrocarbons (PAHs) and polychlorinated biphenyls (PCBs) have the highest detection and reporting frequencies. Researchers $[8,9,27]$ and the International Pellet Watch Program [28] chose PAHs and PCBs as the most important monitoring chemicals because of their relatively high potential to cause adverse health effects. However, few investigations focused on dioxin-like PAHs or PCBs, whose congeners have similar toxic effects as dioxins (2,3,7,8-tetrachlorodibenzo-p-dioxin (TCDD)), are environmentally hazardous and carcinogenic even in small amounts. Effects of such dioxin-like chemicals (DLCs) include thymic atrophy, hepatotoxicity, certain types of cancer, immunotoxicity, and reproductive toxicity [29]. Additionally, DLCs are coplanar molecules, which have relatively higher sorption affinities toward plastics [30]; therefore, more attention should be directed toward the adverse effects of DLCs associated with microplastics.

A number of studies have demonstrated that many of the toxic effects from dioxin-like PAHs and PCBs are mediated via the aryl hydrocarbon receptor (AhR) [31]. The AhR binds co-planar aromatic compounds with high affinity and translocates them into the nucleus [29]. However, it is still not clear at present whether DLCs on microplastics will pose a threat to organisms, or if microplastic retention time in the environment will change the fate and toxicity of the associated

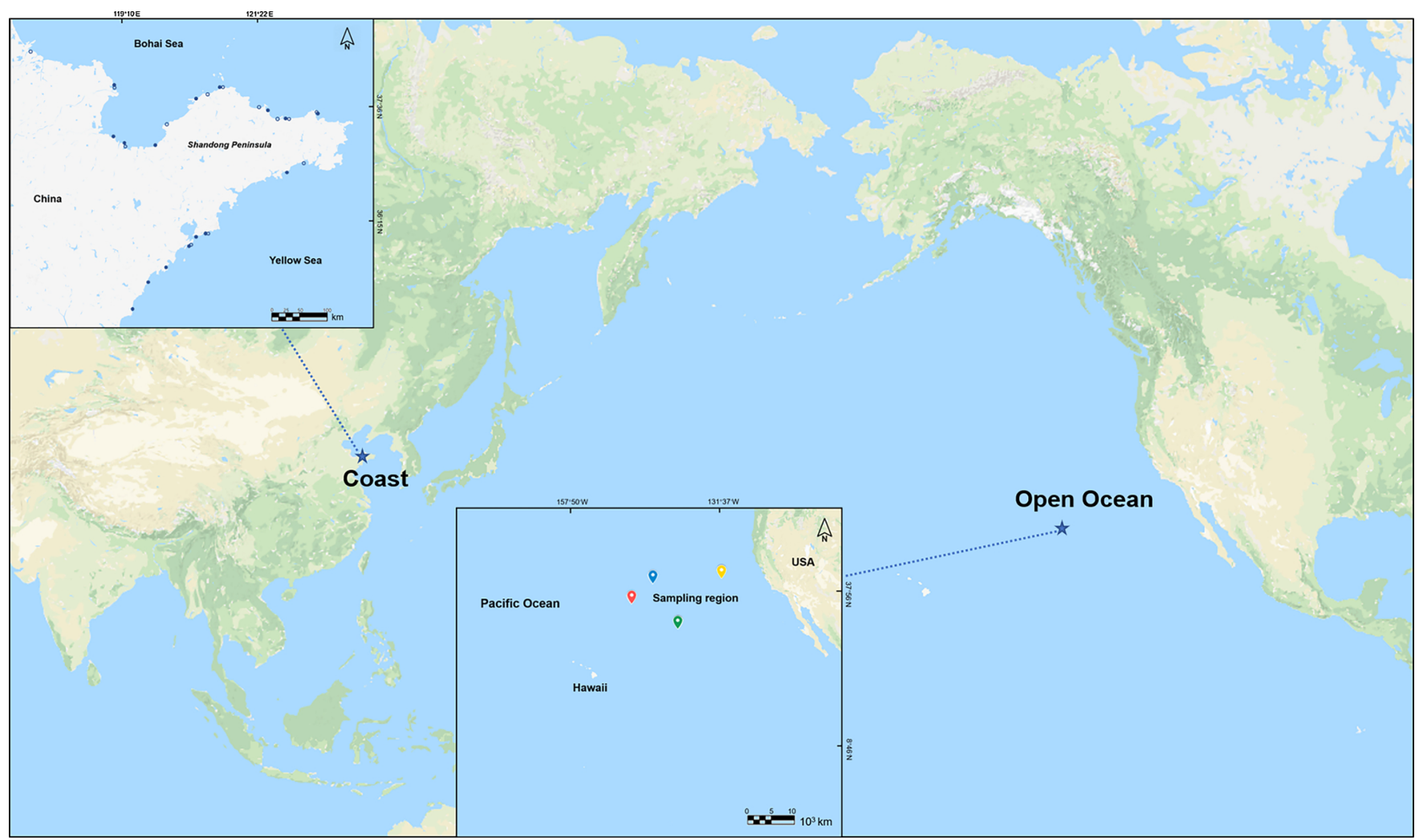

Fig. 1. Marine plastic particles were collected from coastal and open ocean areas. Coast: coastal sampling zones were around Shandong Peninsula, East China; solid circles: styrofoam sampling locations; empty circles: pellet sampling locations. Open Ocean: open ocean sampling areas were within the North Pacific Subtrophic Gyre; the sampling region were located between Hawaii and California, USA. 
DLCs. One of the main reasons of uncertainty is that details about the exposure of microplastic bound DLCs at environmentally relevant concentrations are quite limited.

In the present study, we compared concentrations of dioxin-like PAHs and PCBs in plastic debris from two plastic accumulation zones in marine environments. One accumulation zone was located in Shandong Peninsula (North Pacific Coastal zones; high plastic debris density and high human activity due to laminaria and sea cucumber cultures) and the other was located in the open ocean (North Pacific plastic accumulation zone; high plastic debris density and low human activity). The collected plastic particles included different polymer types (polyethylene (PE), polypropylene (PP) and polystyrene (PS)), and different aging time (unaged and aged microplastics). The objectives of the present study were to: (1) examine the effects of marine regions, polymer types, and aging effects on microplastic bound DLCs; (2) assess the associated DLC health risks based on chemical quantification, micro- ethoxyresorufin- $O$-deethylase (micro-EROD) bioassays and predicted dioxin-like effects to marine organisms through either aqueous or dietary exposures.

\section{Experimental}

\subsection{Sample collection and preparation}

Coastal microplastics samples were collected from the beaches of Shandong Peninsula, East China using a clean stainless-steel shovel from the top $2 \mathrm{~cm}$ profiles in the intertidal zone between the waterline and high tide line. Open ocean plastic samples were collected in the North Pacific Accumulation Zone by manta trawl with an opening mouth of $15 \mathrm{~cm} \times 90 \mathrm{~cm}$ and a mesh size of $500 \mu \mathrm{m}$ (Fig. 1). The plastic samples were grouped as shown in Fig. S1 with different marine regions, sizes, polymer types, and aging time. In each sampling site, we could only collect a few plastic particles, thus we pooled all collected plastic particles together for coastal and open oceanic regions, respectively. Then, we separated the coastal samples into 3 groups of UA pellet (unaged pellets), A pellet (aged pellets), styrofoam (styrofoams); and we also separated the open oceanic samples into 3 groups consisting of small (0. 5-1.5 mm hard plastics), medium (1.5-5 mm hard plastics), and large (5-15 mm hard plastics). Aged/unaged pellets were defined according to Endo et al. [26]: aged pellets were discolored pellets with a visible yellowness of 40 or more when they were laid on a white disposable towel under a fluorescent lamp, while unaged pellets were clear, opaque white or grey pellets. We performed procedural blanks which were conducted all the same in the field and lab processes only without plastics to ensure the operation reliability. Besides, we also prepared virgin commercial PE, PP, and PS plastic particles by cutting purchased plastic thick sheets into $1.5-5 \mathrm{~mm}$ particles (Alfa Aesar, Germany). Further information and details about the extraction procedures can be found in the SI (See S2.1, Table S1).

\subsection{Identification of marine plastic polymer composition}

Among the hundreds of marine plastic particles collected, twenty out of each sampling group were randomly selected for polymer composition identification via micro-Fourier Transform Infrared Spectroscopy (Bruker, LUMOS FTIR Microscope, UK) [32]. The details can be found in SI (See S2.2).

\subsection{Quantification of PAHs and PCBs}

The marine plastic samples were extracted, purified and analyzed for sixteen PAHs, including 7 dioxin-like PAHs (dl-PAHs) of benzo[a] anthracene (BaA), chrysene (CHR), benzo[b]fluoranthene (BbF), benzo [k]fluoranthene $(\mathrm{BkF})$, benzo[a]pyrene (BaP), indeno[1,2,3-cd]pyrene (InP), and dibenz [a,h]anthracene (DbahA); and 9 other PAH congeners (naphthalene (NAP), acenaphthylene (ACY), acenaphthene (ACE), fluorene (FLR), phenanthrene (PHE), anthracene (ANT), fluoranthene (FLN), pyrene (PYR), benzo[g,h,i]perylene (BghiP)). Eighteen measured PCB congeners were comprised of 8 dioxin-like PCBs (CB-77, CB81, CB-105, CB-114, CB-118, CB-126, CB-156, CB-169) and 10 other PCB congeners (CB-28, CB-52, CB-101, CB-123,CB-138, CB-153, CB157, CB-167, CB-180, CB-189) according to Clemons et al. [33] for in vitro rat liver cell lines bioassays. The details about the methods of quantification and limitations can be found in SI (See S2.3).

\subsection{Neutral red assay}

The neutral red uptake assay was performed to estimate the cytotoxicity of extracts prior to conducting the micro-EROD bioassays according to previous methods [34,35]. Experimental details can be found in SI (See S2.4).

\subsection{Micro-EROD assay}

A well-documented effect of DLCs is the aryl hydrocarbon receptor (AhR)-mediated induction of cytochrome P450 enzymes such as the CYP1A-dependent monooxygenases [36]. This mechanism is examined in the micro-EROD assay and deployed in the present work to assess (micro/meso) plastic-associated substances for dioxin-like effects [37]. Experimental details can be found in SI (See S2.5).

\subsection{Bioanalytical equivalent quantification and comparison}

Considering the complexity of organic pollutants and the relatively low concentrations of each chemical on marine environmental plastic particles, concentration addition mechanism has been suggested as a conservative approach to evaluate the mixture toxicity of the associated pollutants [38]. Here, we adopted the bioanalytical equivalent quotient (BEQ) concept [39] to determine the contribution of detected DLCs to the micro-EROD biological effects, by comparing the BEQ from bioanalysis (BEQbio) and from chemical analysis (BEQchem) [40]. According to the bioassay responses, BEQbio in the present study can be calculated as follows.

$B E Q_{b i o}=B_{i o T E Q_{25}}[p g / g]=\frac{E C_{25} T C D D[p g / m L]}{E C_{25} \text { sample }[\mathrm{g} / \mathrm{mL}]}$

BEQchem values of DLCs are calculated as Eq. (2):

$B E Q_{\text {chem }}=\sum_{i=1}^{n} R E P_{i} \cdot C_{i}$

where REPi represents the relative equivalent potency (REP) of the detected DLCs [41]. REP values for DLCs can be found in Table S2.

\subsection{Statistical analysis}

The data analysis in the present study was processed using SPSS (version 22.0). PAH and PCB concentration data among different sampling locations, sizes, polymer types, and ages were first tested for normality and homogenous variance and transformations (log) were applied when needed. Subsequently one-way ANOVA tests or t tests were conducted when parametric assumptions were met; otherwise non-parametric multivariate rank tests were conducted.

\section{Results and discussion}

\subsection{Elevated PAH concentrations on coastal plastics}

The PAH concentrations were significantly greater in plastic particles from coastal beaches $(1722.9-31764.8 \mu \mathrm{g} / \mathrm{kg})$ than in plastic particles from open ocean areas (nd-6298.8 $\mu \mathrm{g} / \mathrm{kg}$ ) (Fig. S2A; $p=0.034$; $\mathrm{nd}=$ not detectable, which represents values $<$ limit of quantification 
(LOQ)). As for dioxin-like PAHs (dl-PAHs), the concentration ranges were 5.2-9170.3 and nd-13226.2 ng TCDD/g plastic for coastal and oceanic plastics, respectively (Fig. $2 \mathrm{~A}$ purple dots; $p=0.076$ ). A previous study reported PAH concentrations on microplastics within the same coastal region close to Shandong Peninsular ranged from 136.3 to $1586.9 \mu \mathrm{g} / \mathrm{kg}$ and from 397.6 to $2384.2 \mu \mathrm{g} / \mathrm{kg}$ [42], which is comparable to the PAH concentrations from coastal regions observed in the present study. The greater PAH concentrations observed in our study were likely because the Shandong Peninsula coast is located near industrialized areas and urban beaches are closer to pollution sources $[43,44]$. The elevated concentrations of PAHs on coastal plastics are likely to be the result of sorption from the surrounding environment, because PAHs have a strong affinity to plastic particles [19], with $K_{d}$ values of 5.6-7.0 for dioxin-like PAHs on PE [45].

Furthermore, it is noteworthy to state that styrofoam microplastics exhibited particularly greater PAHs/dl-PAHs than or the other plastic particles, which had been marked with highlighted red background colour in Fig. 2A. This was probably because that PS plastic functioned as a sink for some kinds of PAHs. Rochman et al. [46] reported that seven non-dioxin-like parent PAHs of ACY, ACE, FLR, PHE, ANT, FLN and PYR presence on virgin PS plastics from 5.2 to $25.4 \mu \mathrm{g} / \mathrm{kg}$ plastic. But we did not detect PAHs in our virgin PS plastics which was probably due to different production engineering (Table S3). Another explanation was that styrofoam is composed of expanded PS, which had greater sorption ability for PAHs if compared with PE or PP plastics due to more amorphous regions on styrofoams [47]. Detailed discussion will be carried out in Section 3.3.2.

\subsection{Concentrations of PCBs on coastal and oceanic plastics}

Concentrations of sum PCBs detected on the plastic particle samples did not show significant location differences between coastal areas $(68.9-261.4 \mu \mathrm{g} / \mathrm{kg}$ ) and open ocean (nd-694.0 $\mu \mathrm{g} / \mathrm{kg}$ ) among all sizes of plastic particles (Fig. S2B; $p=0.40$ ). In contrast, dioxin-like PCBs (dlPCBs) were higher in coastal areas than those in open ocean, with relative equivalent BEQchem values of $0-4996.6$ and $24.8-507.6 \mathrm{pg}$
$\mathrm{TCDD} / \mathrm{g}$ plastic, respectively (Fig. 2B purple dots; $p=0.27$ ). As for open ocean plastics, a previous study of ours reported PCB concentrations in North Pacific subtropical regions $(0.7-308.4 \mu \mathrm{g} / \mathrm{kg}$ ) [8] that were lower than those of the present study for the same type of plastic particles within the same region. Differences in PCB concentrations between studies can be attributed to the measurement of different PCB congeners.

Coplanar PCB congeners (the dominant PCB congeners in the present study showed lower photoreactions than other PCB congeners, therefore preventing their photolysis under sunlight and ultraviolet light due to increased electron affinity with the extended conjugation between two phenyl rings in the planar configuration [48]. The high detection frequency for CB-77 (32\%) (Fig. 2B) in open ocean plastic samples also suggests the potential occurrence of photodegradation. The degradation product of many dl-PCB congeners (CB-105, 118, 126, 169) have a high yield of CB-77 after photolysis [48,49]. Moreover, dlPCBs can even be generated from some potential precursor congeners during photodegradation, which are very abundant in commercial PCB mixtures (e.g., Aroclor products) [48]. Thus, CB-77 was detected in all coastal plastics (Fig. 2B), largely due to CB-77 formation during hightemperature processes [50] and photodegradation that would typically occur on the shore $[48,49]$. In the coastal region, dl-PCBs seldom exist naturally, but they can be detected in some industrial products or formed through combustion [50]. PCBs tend to accumulate in the organic phase of sediments [51], thus, it is not surprising that we also observed elevated concentrations of dl-PCBs on coastal plastics, which follow the same partitioning mechanisms for PCBs as sediments [52].

\subsection{The influence of plastic size, type, and aging}

\subsubsection{Plastic particle size}

We tested the size effect of plastic particles on pollutants associated with open ocean plastics (Fig. 2). PAH concentrations nd-6.2, nd6298.8 , and nd- $8.3 \mu \mathrm{g} / \mathrm{kg}(p=0.323)$ and PCB concentrations nd210.1, nd-412.4, nd-694.0 $\mu \mathrm{g} / \mathrm{kg}(p=0.4193)$ did not significantly differ among small, medium and large particle sizes, respectively. This
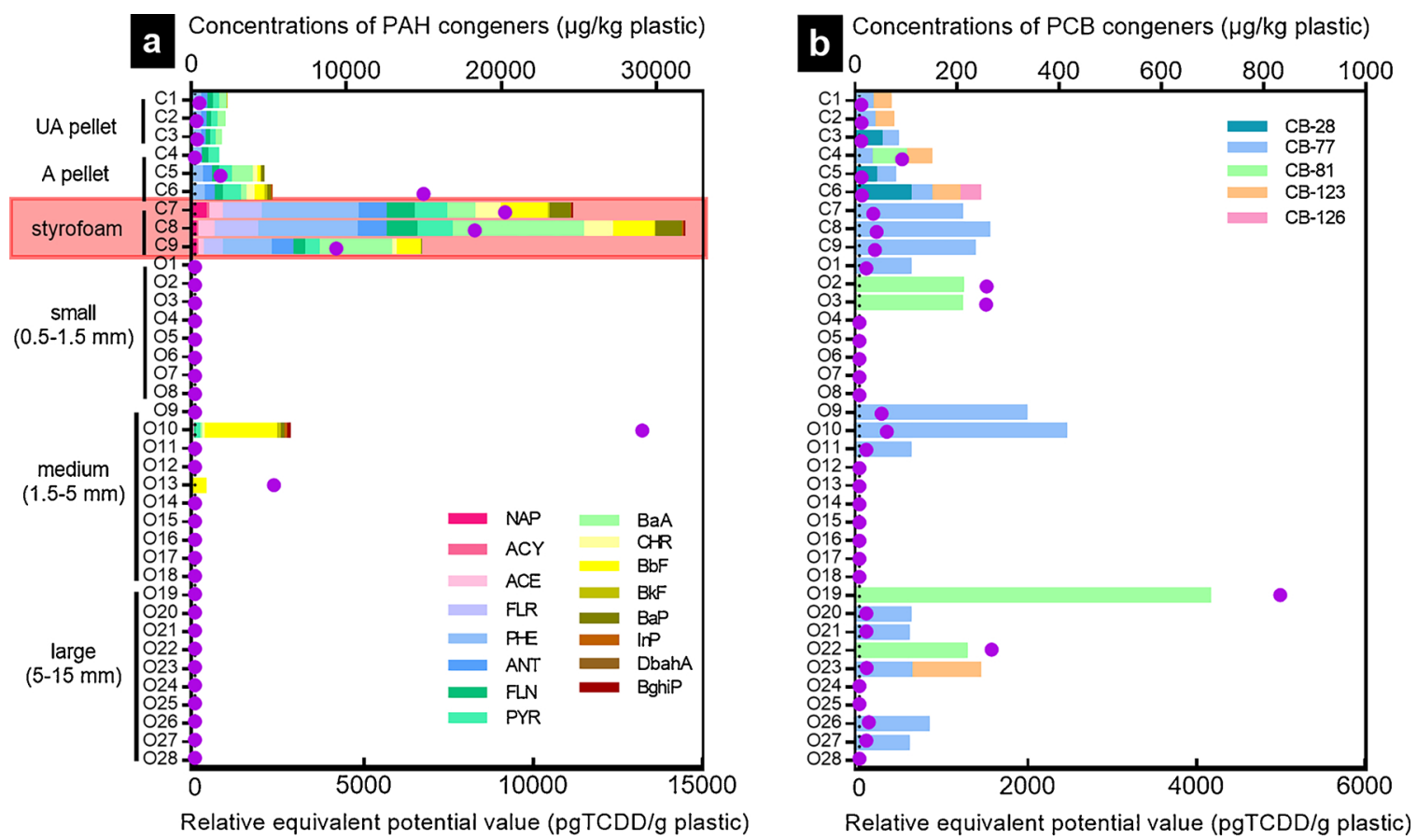

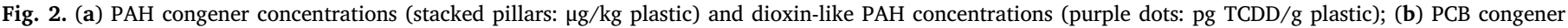

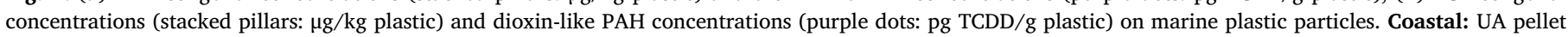

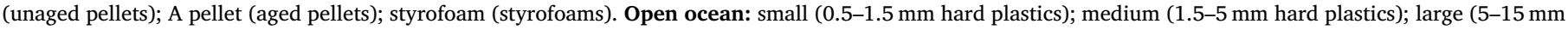
hard plastics) (For interpretation of the references to colour in this figure legend, the reader is referred to the web version of this article). 
result suggests that pollutant concentrations versus particle size did not occur, which is likely due to the partition sorption mechanisms of PAHs and PCBs onto plastics $[8,53]$. Similar to the present study, no effect of the particle size $(0.5 \mathrm{~mm}-150 \mathrm{~mm})$ was also previously observed for other HOCs (such as polybrominated diphenyl ethers and hexabromocyclododecane) that we measured on plastic debris from the North Pacific Open Ocean [8]. Under non-equilibrium conditions, smaller particles possess a shorter intrapolymer diffusion path length than larger ones, which may lead to HOC concentration dependence on size. However, Koelmans et al. [23] predicted that $80-90 \%$ of plastic debris present in the ocean are older than 2-4 years, a time length that ensures the majority of HOCs achieve or are close to sorption equilibrium. Additionally, another explanation is that the plastic particle samples examined in the present study were all in the millimeter-size range. According to our best knowledge, it has been predicted that plastic particles with smaller sizes had a higher surface area for sorption than larger particles [54], and our unpublished data found that polyethylene plastic $(\mu \mathrm{m}-\mathrm{mm})$ sorption ability for antibiotics increased with decreasing plastic sizes. Thus, the effect of size may need to be verified further by accounting for micrometer- or even nanometer-sized plastics in future studies.

\subsubsection{Plastic polymer type}

Polymer type showed significant influence on HOC concentrations on plastics in the coastal region. Concentrations of organic pollutants on styrofoam (PS, $\quad \Sigma$ PCBs $=234.6 \pm 26.1 \mu \mathrm{g} / \mathrm{kg}, \quad \Sigma \mathrm{PAHs}=$ $23,696.7 \pm 8516.3 \mu \mathrm{g} / \mathrm{kg}$ ) were much greater than those in unaged and aged pellets (PE/PP, $\quad \Sigma$ PCBs $=116.0 \pm 69.2 \mu \mathrm{g} / \mathrm{kg}$, $\Sigma \mathrm{PAHs}=2958.2 \pm 1522.1 \mu \mathrm{g} / \mathrm{kg})(p=0.027$ for PAHs; $p=0.024$ for PCBs). A similar phenomenon was also observed by Lee et al. [18], who found that the partitioning coefficients between different types of microplastics and concentrations of PAHs and PCBs in seawater were generally in the order of PS > PE > PP. Meanwhile, Rochman et al. [46] also found that PS behaved as a sink for parent PAHs, with 8-200 times greater sorption of PAH concentrations on PS than on polyethylene terephthalate (PET), polyvinyl chloride (PVC), PE and PP. Greater sorption on PS may be attributed to the different degree of crystallinity of these polymers, which usually contain crystalline and amorphous regions [47]. PP, high-density polyethylene (HDPE) and low-density polyethylene (LDPE) can be classified as semi-crystal polymers with $70-80 \%, 70-80 \%$, and $45-55 \%$ crystallinity, respectively [55].The crystal region within the polymers have regularly arranged atoms in terms of positions and motions. Therefore, pollutants do not favorably absorb to crystalline regions because a high energy is required to disrupt tightly associated polymeric chains [47]. However, PS is an amorphous polymer, where polymeric chains are more randomly oriented and a larger free volume is available for organic chemicals to move into and be absorbed [47]. An additional explanation for greater concentrations of pollutants on styrofoam is that styrofoam has larger surface area for contact with surrounding seawater per unit mass. The density of expanded PS (styrofoam) is $0.01-0.04 \mathrm{~g} / \mathrm{cm}^{3}$, largely lower than PE $\left(0.89-0.98 \mathrm{~g} / \mathrm{cm}^{3}\right)$ and PP $\left(0.83-0.92 \mathrm{~g} / \mathrm{cm}^{3}\right)$ [56]. Although there were contrasting results for the PS sorption capacity [57], the lowered PS sorption ability was more likely due to the different polymer composition produced by different manufacturers.

\subsubsection{Plastic aging effect}

For coastal microplastics, we compared the aging effect on coastal plastic burdens of PAHs and PCBs. The microplastic pellets showed differences to some extent with regard to the influence of aging (Fig. 2). In particular, FLN, PYR and BghiP significantly increased their concentrations during the aging time $(p<0.05)$ (Table S3). One explanation may be that the increased occurrence of PAH congeners had high background sedimentary concentrations reported in the same coastal area [58], and thus these pellets undergoing aging processes were exposed to, and further sorb various PAHs, in the coastal area during their longer residence time. Therefore, the aging process could influence the PAH composition and concentration on coastal microplastics, which would also subsequently alter their potential ecotoxicity.

\subsection{Integrated micro-EROD bioanalysis and chemical analysis}

Due to high hydrophobicity and large surface area to volume ratio, microplastics can contain a variety of organic micropollutants, and therefore the screened DLCs may only be a small fraction of the
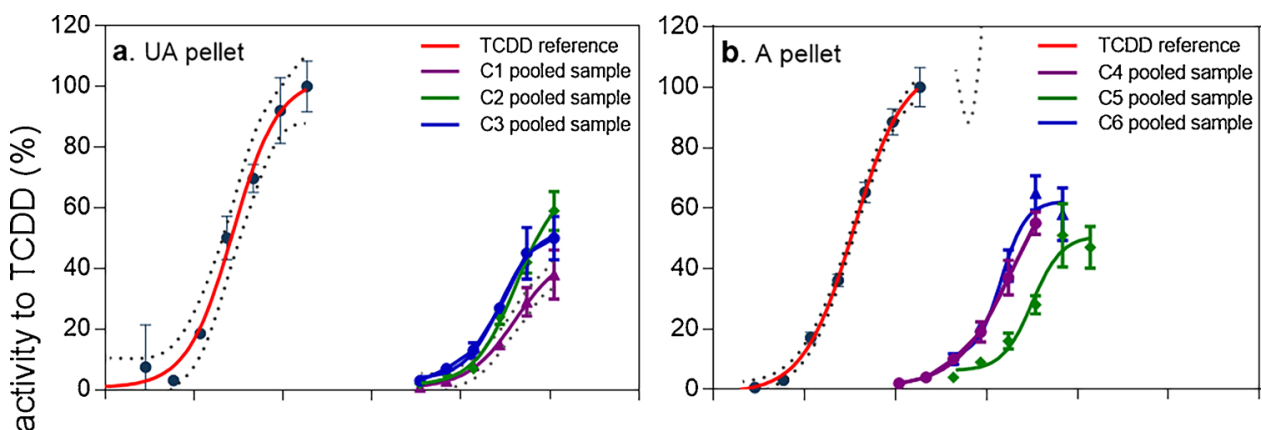

Fig. 3. The micro-EROD bioassay results for coastal plastic particles extracts of (a) UA pellet (unaged pellets), (b) A pellet (aged pellets), (c) styrofoams, and (d) diluted styrofoam samples. The red curve is the micro-EROD activity curve of standard reference of TCDD (2,3,7,8-tetrachorodibenzodioxin); the green, purple, and blue curves were relative microEROD activities to TCDD for sample extractions. (For interpretation of the references to colour in this figure legend, the reader is referred to the web version of this article).

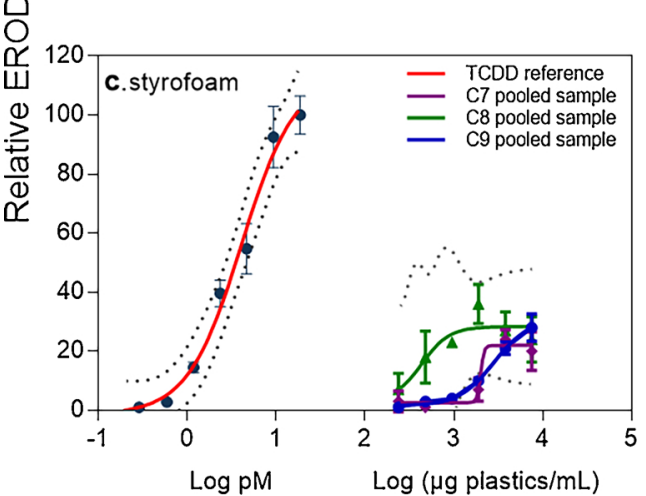

Log pM

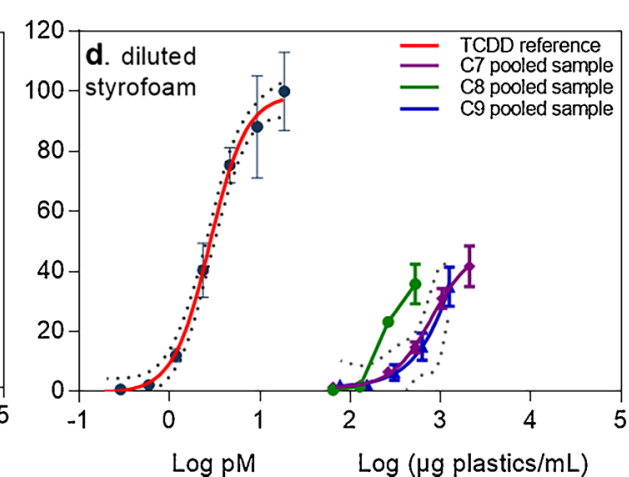


chemicals present on microplastics. Based on this, the micro-EROD bioassay can be a valuable analysis to targeted chemical quantification, which provides comprehensive conclusions about AhR activation effects.

Overall, we found that the BEQ model was suitable to investigate the dioxin-like effects driven by DLCs on microplastic pellets by assuming an addition mixture toxicity mechanism [39]. The calculated BEQbio values were 219-260 and 2269-6070 pgTCDD/g plastic for unaged pellet and aged pellet respectively, according to micro-EROD bioassays (Fig. 3a \&b). By comparing BEQbio and BEQchem values (Fig. $4 \mathrm{a} \& \mathrm{~b}$ ), it was found that the unaged and aged pellets exhibited high induction for the AhR activated dioxin-like effects, and with relative good chemical-biological causal explanation of $37-73 \%$ and $11-112 \%$ for unaged and aged pellets, respectively (Table S4). The AhR effect was dominantly driven by $\mathrm{BbF}$ and $\mathrm{BkF}$ chemicals for both aged and unaged pellets, whereas, dioxin-like PCB concentrations increased in aged plastics, which may be attributed to the sorption during the prolonged retention time in the marine environment (Fig. 1).

However, for styrofoam samples, it was difficult to determine the contribution of detected chemicals to dioxin-like effects. Although elevated concentrations of BbF and CB-77 were present in extracts of styrofoam samples (Fig. 2), and the total predicted REP values were the highest, the micro-EROD bioassay measured dioxin-like effect in styrofoam samples were lower than that of the aged pellet extracts (Fig. 3c), thus abnormally high chemical contribution percentages for styrofoam samples were obtained (114-534\%, Table S4).

We further explored the underlying reasons for the abnormally low activation of micro-EROD activity for styrofoam samples (Fig. 3c). One reason is likely due to the lowered bioavailability of DLCs in the styrofoam samples. Partial PS molecules can be dissolved during extraction with dichloromethane (DCM), because PS possesses polarizable side groups and can be dissolved by aromatics or strongly polar groups containing solvents, such as DCM [59]. Therefore, DCM molecules have favorable interactions with PS. Additionally, styrene polymer segments may be present in solution and sorb DLCs, subsequently decreasing their bioavailability to exposed cells. On the other hand, styrene oligomers, such as styrene trimers can be present in the extracts, which have previously been reported to be derived from PS degradation [60]. Styrene is known to have three aromatic rings, which is structurally similar to AhR-active compounds [61], and Hong et al. [62] demonstrated that the REPs of styrene were 1000- to 10,000- fold less than $\mathrm{BaP}$. Due to the large amounts of styrene trimmers, competition with other DLC molecules may exist due to the occupation of binding positions.

In order to verify this, we diluted the original styrofoam extracts by 1000 folds, which can alleviate the competition status for Ah receptor. Then we measured the diluted styrofoam extraction samples with micro-EROD bioassays, and found that much higher micro-EROD activity induction effects were observed for the diluted samples (Fig. 3d), and subsequently much better linkage between chemical analysis (BEQchem) and biological signals (BEQbio) were also observed after dilution (Fig. 4c) with an alleviation of the competition by styrene oligomers (Fig. 4d).

\subsection{Potential dioxin-like effects on marine organisms}

In order to simulate actual scenarios, we predicted the DLC concentrations desorbed from microplastics to marine organisms through either aqueous or dietary exposure ways. According to the literature, the releasing percentages of organic contaminants from microplastics were from lower than $1 \%$ in seawater to $78 \%$ in simulated gut surfactants of $15 \mathrm{mM}$ sodium taurocholate (ST) (Table 1) [63,64]. Although
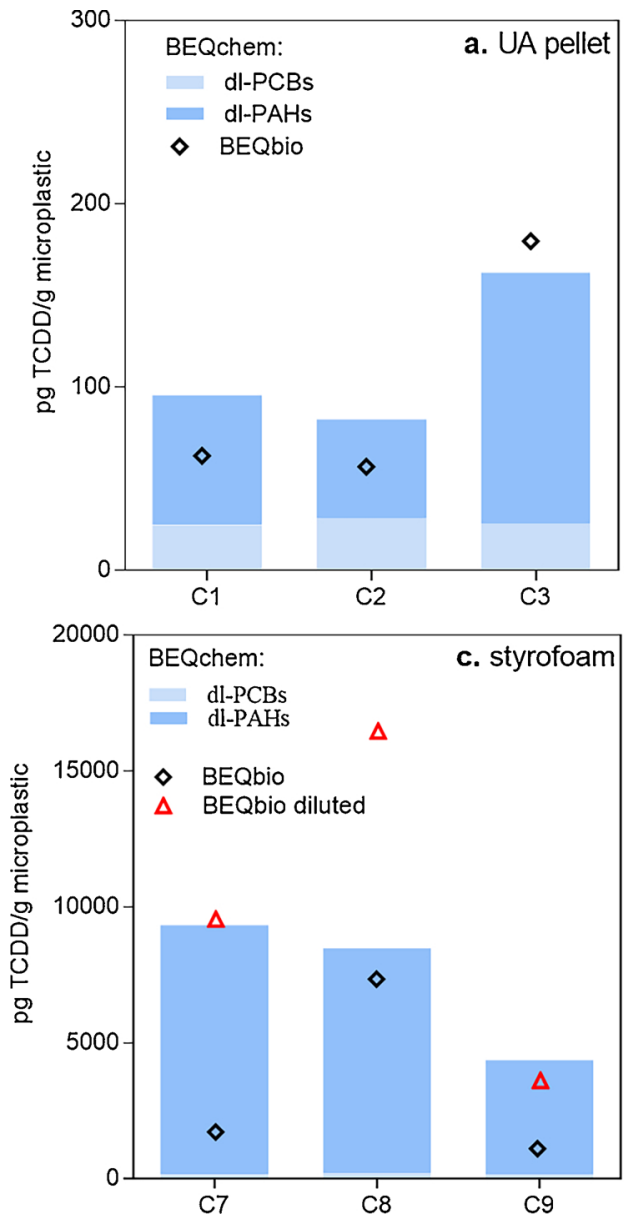
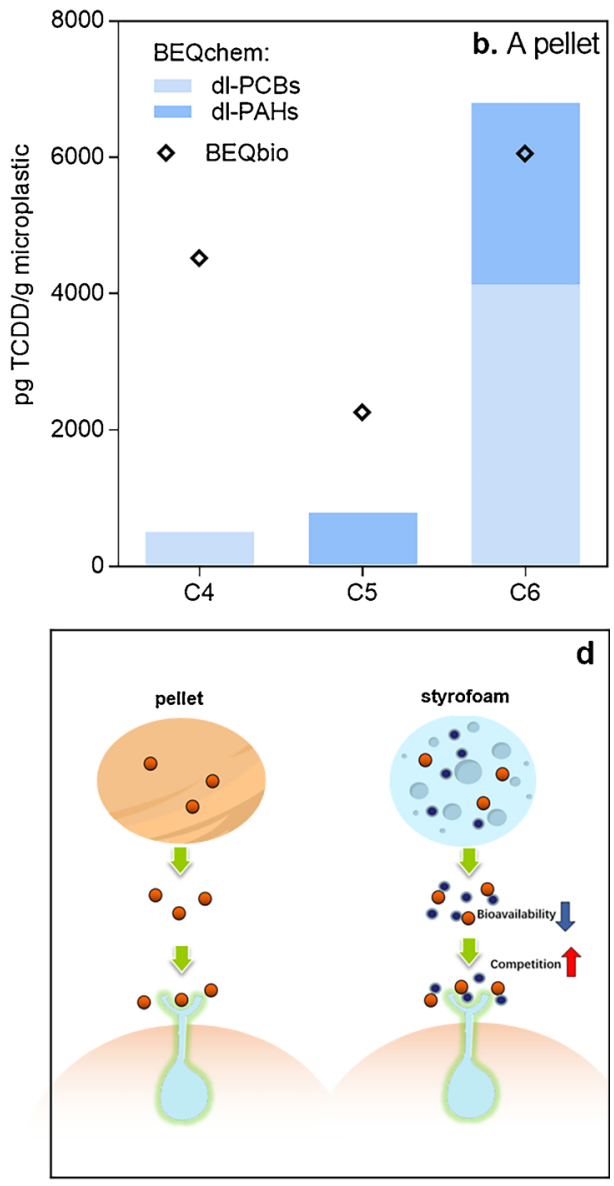

Fig. 4. Contribution percentages of the measured concentrations of DLCs to the dioxin-like effects determined according to micro-EROD bioassay for (a) UA pellet (unaged pellets); (b) A pellet (aged pellets). BEQchem: the calculated relative TCDD concentrations according to chemical analysis; BEQbio: the measured relative TCDD concentrations based on microEROD bioassays. (c) styrofoams; (d) a sketch of the possible mechanisms of micro-EROD inhibition for styrofoam extraction to receptors. Styrene oligomers can sorb DLCs to decrease their bioavailability, and they can also be agonists to compete with other DLCs for aryl hydrocarbon-receptors (AhR). 


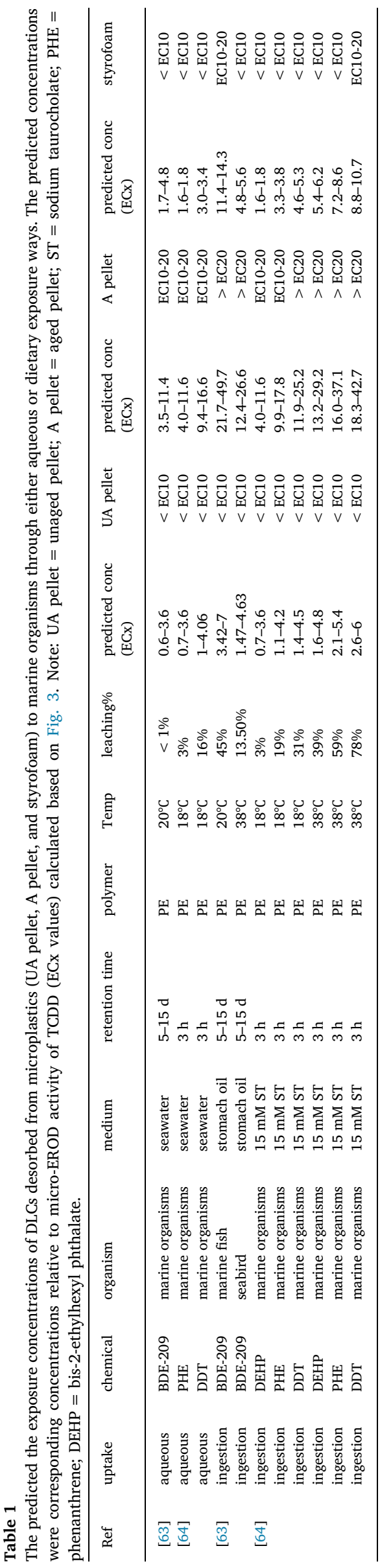

the compounds (i.e., BDE-209, PHE, DDT, and bis-2-ethylhexyl phthalate (DEHP)) tested $[63,64]$ were not all the same as those measured in the present study, their logarithm of octanol water distribution coefficients (logKow) (4.46-9.97) were quite similar.

For pollutants on unaged pellets, all scenarios exhibit effects below EC10 thresholds, which suggests that dioxin-like effects may not occur. This is because that the EC10 can replace the No Observed Effect Concentration (NOEC), which is a commonly used effect parameter in microbial tests [65]. However, the results suggest that particular attention should be given to aged pellets and styrofoams. Their predicted leaching DLC concentrations have exceeded EC10 thresholds with $100 \%$ ( 11 out of 11 scenarios) for aged pellets and $18 \%$ ( 2 out of 11 scenarios) for styrofoams, implying that dioxin-like effects are likely to occur once these microplastics exposed to marine organisms.

\section{Conclusions}

In conclusion, the concentrations of DLCs on plastic particles are mainly influenced by coastal/oceanic regions, polymer types, and aging effects, but not plastic size. Chemical analysis demonstrated that coastal styrofoams possessed the greatest DLC concentrations, because of the presence of additives and greater sorption ability relative to other plastics (PP and PE). However, the comprehensive micro-EROD bioassay results indicated a relative lower dioxin-like effect for styrofoam samples. The discrepancies could be attributed to the decreased DLC bioavailability and increased receptor binding competition in the presence of styrene oligomers. Furthermore, aged pellets and styrofoams have been observed to have potential dioxin-like risks to marine organisms through either aqueous or dietary exposure scenarios. While DLCs bound on marine microplastics are not yet reported according to our best knowledge, this work demonstrates that it is an important consideration in risk assessment because they can pose strong health risks to organisms, especially when considering their light density and easy to transport behavior in the marine environment.

\section{Acknowledgements}

We appreciate the oceanic plastics donation by The Ocean Cleanup Foundation and CSC/DAAD postdoc fellowship to Dr. Qiqing Chen during her stay in Germany. This project was supported by National Natural Science Foundation of China (41771351), Shanghai Pujiang Talent Program (18PJ1403400), and East China Normal University Independent Project (SKLEC-2018RCDW01), Zhejiang A\&F University Research Funding (2017FR021).

\section{Appendix A. Supplementary data}

Supplementary material related to this article can be found, in the online version, at doi:https://doi.org/10.1016/j.jhazmat.2018.10.032.

\section{References}

[1] L. Yin, B. Chen, B. Xia, X. Shi, K. Qu, Polystyrene microplastics alter the behavior, energy reserve and nutritional composition of marine jacopever (Sebastes schlegelii), J. Hazard. Mater. 360 (2018) 97-105.

[2] A.L. Andrady, Microplastics in the marine environment, Mar. Pollut. Bull. 62 (2011) 1596-1605.

[3] J. Lee, J.S. Lee, Y.C. Jang, S.Y. Hong, W.J. Shim, Y.K. Song, S.H. Hong, M. Jang, G.M. Han, D. Kang, S. Hong, Distribution and size relationships of plastic marine debris on beaches in South Korea, Arch. Environ. Con. Tox. 69 (2015) 288-298.

[4] O.S. Alimi, J.F. Budarz, L.M. Hernandez, N. Tufenkji, Microplastics and nanoplastics in aquatic environments: aggregation, deposition, and enhanced contaminant transport, Environ. Sci. Technol. 52 (2018) 1704-1724.

[5] A.L. Dawson, S. Kawaguchi, C.K. King, K.A. Townsend, R. King, W.M. Huston, S.M.B. Nash, Turning microplastics into nanoplastics through digestive fragmentation by Antarctic krill, Nat. Commun. 9 (2018).

[6] R.C. Thompson, Y. Olsen, R.P. Mitchell, A. Davis, S.J. Rowland, A.W. John, Lost at sea: where is all the plastic? Science 304 (2004) 838.

[7] C.M. Rachman, Microplastics research - from sink to source, Science 360 (2018) 28-29. 
[8] Q.Q. Chen, J. Reisser, S. Cunsolo, C. Kwadijk, M. Kotterman, M. Proietti, B. Slat, F.F. Ferrari, A. Schwarz, A. Levivier, D.Q. Yin, H. Hollert, A.A. Koelmans, Pollutants in plastics within the north pacific subtropical gyre, Environ. Sci. Technol. 52 (2018) 446-456.

[9] L.M. Rios, P.R. Jones, C. Moore, U.V. Narayan, Quantitation of persistent organic pollutants adsorbed on plastic debris from the Northern Pacific Gyre's "eastern garbage patch", J. Environ. Monitor. 12 (2010) 2226-2236.

[10] R.C. Hale, Are the risks from microplastics truly trivial? Environ. Sci. Technol. 52 (2018) 931-931.

[11] H. Qu, R. Ma, B. Wang, Y. Zhang, L. Yin, G. Yu, S. Deng, J. Huang, Y. Wang, Effects of microplastics on the uptake, distribution and biotransformation of chiral antidepressant venlafaxine in aquatic ecosystem, J. Hazard. Mater. 359 (2018) 104-112.

[12] J.N. Hahladakis, C.A. Velis, R. Weber, E. Iacovidou, P. Purnell, An overview of chemical additives present in plastics: migration, release, fate and environmental impact during their use, disposal and recycling, J. Hazard. Mater. 344 (2018) 179-199.

[13] Q.Q. Chen, M. Gundlach, S.Y. Yang, J. Jiang, M. Velki, D.Q. Yin, H. Hollert, Quantitative investigation of the mechanisms of microplastics and nanoplastics toward zebrafish larvae locomotor activity, Sci. Total Environ. 584 (2017) 1022-1031.

[14] Q.Q. Chen, D.Q. Yin, Y.L. Jia, S. Schiwy, J. Legradi, S.Y. Yang, H. Hollert, Enhanced uptake of BPA in the presence of nanoplastics can lead to neurotoxic effects in adult zebrafish, Sci. Total Environ. 609 (2017) 1312-1321.

[15] M. Oliveira, A. Ribeiro, K. Hylland, L. Guilhermino, Single and combined effects of microplastics and pyrene on juveniles ( $0+$ group) of the common goby Pomatoschistus microps (Teleostei, Gobiidae), Ecol. Indic. 34 (2013) 641-647.

[16] C.M. Rochman, T. Kurobe, I. Flores, S.J. Teh, Early warning signs of endocrine disruption in adult fish from the ingestion of polyethylene with and without sorbed chemical pollutants from the marine environment, Sci. Total Environ. 493 (2014) $656-661$.

[17] F. Gallo, C. Fossi, R. Weber, D. Santillo, J. Sousa, I. Ingram, A. Nadal, D. Romano, Marine litter plastics and microplastics and their toxic chemicals components: the need for urgent preventive measures, Environ. Sci. Eur. 30 (2018) 13.

[18] H. Lee, W.J. Shim, J.H. Kwon, Sorption capacity of plastic debris for hydrophobic organic chemicals, Sci. Total Environ. 470 (2014) 1545-1552.

[19] E.L. Teuten, J.M. Saquing, D.R.U. Knappe, M.A. Barlaz, S. Jonsson, A. Bjorn, S.J. Rowland, R.C. Thompson, T.S. Galloway, R. Yamashita, D. Ochi, Y. Watanuki, C. Moore, H.V. Pham, T.S. Tana, M. Prudente, R. Boonyatumanond, M.P. Zakaria, K. Akkhavong, Y. Ogata, H. Hirai, S. Iwasa, K. Mizukawa, Y. Hagino, A. Imamura, M. Saha, H. Takada, Transport and release of chemicals from plastics to the environment and to wildlife, Philos. Trans. Biol. Sci. 364 (2009) 2027-2045.

[20] K.L. Law, R.C. Thompson, Microplastics in the seas, Science 345 (2014) 144-145.

21] R.C. Thompson, C.J. Moore, F.S. vom Saal, S.H. Swan, Plastics, the environment and human health: current consensus and future trends, Philos. Trans. Biol. Sci. 364 (2009) 2153-2166.

[22] M. Cole, P. Lindeque, C. Halsband, T.S. Galloway, Microplastics as contaminants in the marine environment: a review, Mar. Pollut. Bull. 62 (2011) 2588-2597.

[23] A.A. Koelmans, A. Bakir, G.A. Burton, C.R. Janssen, Microplastic as a vector for chemicals in the aquatic environment: critical review and model-supported reinterpretation of empirical studies, Environ. Sci. Technol. 50 (2016) 3315-3326.

[24] K. Tanaka, H. Takada, R. Yamashita, K. Mizukawa, M. Fukuwaka, Y. Watanuki, Accumulation of plastic-derived chemicals in tissues of seabirds ingesting marine plastics, Mar. Pollut. Bull. 69 (2013) 219-222.

[25] C. Zarfl, M. Matthies, Are marine plastic particles transport vectors for organic pollutants to the Arctic? Mar. Pollut. Bull. 60 (2010) 1810-1814.

[26] S. Endo, R. Takizawa, K. Okuda, H. Takada, K. Chiba, H. Kanehiro, H. Ogi, R. Yamashita, T. Date, Concentration of polychlorinated biphenyls (PCBs) in beached resin pellets: variability among individual particles and regional differences, Mar. Pollut. Bull. 50 (2005) 1103-1114.

[27] H. Hirai, H. Takada, Y. Ogata, R. Yamashita, K. Mizukawa, M. Saha, C. Kwan, C. Moore, H. Gray, D. Laursen, E.R. Zettler, J.W. Farrington, C.M. Reddy, E.E. Peacock, M.W. Ward, Organic micropollutants in marine plastics debris from the open ocean and remote and urban beaches, Mar. Pollut. Bull. 62 (2011) 1683-1692.

[28] B.G. Yeo, H. Takada, H. Taylor, M. Ito, J. Hosoda, M. Allinson, S. Connell, L. Greaves, J. McGrath, POPs monitoring in Australia and New Zealand using plastic resin pellets, and International Pellet Watch as a tool for education and raising public awareness on plastic debris and POPs, Mar. Pollut. Bull. 101 (2015) 137-145.

[29] K. Eichbaum, M. Brinkmann, S. Buchinger, G. Reifferscheid, M. Hecker, J.P. Giesy, M. Engwall, B. van Bavel, H. Hollert, In vitro bioassays for detecting dioxin-like activity - application potentials and limits of detection, a review, Sci. Total Environ. 487 (2014) 37-48.

[30] L.J. Liu, R. Fokkink, A.A. Koelmans, Sorption of polycyclic aromatic hydrocarbons to polystyrene nanoplastic, Environ. Toxicol. Chem. 35 (2016) 1650-1655.

[31] H. Olsman, M. Engwall, U. Kammann, M. Klempt, J. Otte, B. van Bavel, H. Hollert, Relative differences in aryl hydrocarbon receptor-mediated response for 18 polybrominated and mixed halogenated dibenzo-p-dioxins and -furans in cell lines from four different species, Environ. Toxicol. Chem. 26 (2007) 2448-2454.

[32] X.Y. Qu, L. Su, H.X. Li, M.Z. Liang, H.H. Shi, Assessing the relationship between the abundance and properties of microplastics in water and in mussels, Sci. Total Environ. 621 (2018) 679-686.

[33] J.H. Clemons, D.G. Dixon, N.C. Bols, Derivation of 2,3,7,8-TCDD toxic equivalent factors (TEFs) for selected dioxins, furans and PCBs with rainbow trout and rat liver cell lines and the influence of exposure time, Chemosphere 34 (1997) 1105-1119.
[34] G. Repetto, A. del Peso, J.L. Zurita, Neutral red uptake assay for the estimation of cell viability/cytotoxicity, Nat. Protoc. 3 (2008) 1125-1131.

[35] T.B. Seiler, A.C. Rastall, E. Leist, L. Erdinger, T. Braunbeck, H. Hollert, Membrane dialysis extraction (MDE): a novel approach for extracting toxicologically relevant hydrophobic organic compounds from soils and sediments for assessment in biotests, J. Soils Sedim. 6 (2006) 20-29.

[36] H. Hollert, M. Durr, H. Olsman, K. Halldin, B. Van Bavel, W. Brack, M. Tysklind, M. Engwall, T. Braunbeck, Biological and chemical determination of dioxin-like compounds in sediments by means of a sediment triad approach in the catchment area of the River Neckar, Ecotoxicology 11 (2002) 323-336.

[37] A. Schiwy, M. Brinkmann, I. Thiem, G. Guder, K. Winkens, K. Eichbaum, L. Nusser, B. Thalmann, S. Buchinger, G. Reifferscheid, T.B. Seiler, B. Thoms, H. Hollert, Determination of the CYP1A-inducing potential of single substances, mixtures and extracts of samples in the micro-EROD assay with H4IIE cells, Nat. Protoc. 10 (2015) 1728-1741.

[38] A. Kortenkamp, Ten years of mixing cocktails: a review of combination effects of endocrine-disrupting chemicals, Environ. Health Perspect. 115 (2007) 98-105.

[39] P.A. Neale, S. Ait-Aissa, W. Brack, N. Creusot, M.S. Denison, B. Deutschmann, K. Hilscherova, H. Hollert, M. Krauss, J. Novak, T. Schulze, T.B. Seiler, H. Serra, Y. Shao, B.I. Escher, Linking in vitro effects and detected organic micropollutants in surface water using mixture-toxicity modeling, Environ. Sci. Technol. 49 (2015) 14614-14624.

[40] C. Di Paolo, R. Ottermanns, S. Keiter, S. Ait-Aissa, K. Bluhm, W. Brack, M. Breitholtz, S. Buchinger, M. Carere, C. Chalon, X. Cousin, V. Dulio, B.I. Escher, T. Hamers, K. Hilscherova, S. Jarque, A. Jonas, E. Maillot-Marechal, Y. Marneffe, M.T. Nguyen, P. Pandard, A. Schifferli, T. Schulze, S. Seidensticker, T.B. Seiler, J. Tang, R. van der Oost, E. Vermeirssen, R. Zounkova, N. Zwart, H. Hollert, Bioassay battery interlaboratory investigation of emerging contaminants in spiked water extracts - towards the implementation of bioanalytical monitoring tools in water quality assessment and monitoring, Water Res. 104 (2016) 473-484.

[41] M. Van den Berg, L.S. Birnbaum, M. Denison, M. De Vito, W. Farland, M. Feeley, H. Fiedler, H. Hakansson, A. Hanberg, L. Haws, The 2005 World Health Organization reevaluation of human and mammalian toxic equivalency factors for dioxins and dioxin-like compounds, Toxicol. Sci. 93 (2006) 223-241.

[42] W.W. Zhang, X.D. Ma, Z.F. Zhang, Y. Wang, J.Y. Wang, J. Wang, D.Y. Ma, Persistent organic pollutants carried on plastic resin pellets from two beaches in China, Mar. Pollut. Bull. 99 (2015) 28-34.

[43] M. Fisner, S. Taniguchi, A.P. Majer, M.C. Bicego, A. Turra, Concentration and composition of polycyclic aromatic hydrocarbons (PAHs) in plastic pellets: implications for small-scale diagnostic and environmental monitoring, Mar. Pollut. Bull. 76 (2013) 349-354.

[44] J.P.G.L. Frias, P. Sobral, A.M. Ferreira, Organic pollutants in microplastics from two beaches of the Portuguese coast, Mar. Pollut. Bull. 60 (2010) 1988-1992.

[45] R. Lohmann, Critical review of low-density polyethylene's partitioning and diffusion coefficients for trace organic contaminants and implications for its use as a passive sampler, Environ. Sci. Technol. 46 (2012) 606-618.

[46] C.M. Rochman, C. Manzano, B.T. Hentschel, S.L.M. Simonich, E. Hoh, Polystyrene plastic: a source and sink for polycyclic aromatic hydrocarbons in the marine environment, Environ. Sci. Technol. 47 (2013) 13976-13984.

[47] C.E.C Jr, Carraher's Polymer Chemistry, Ninth Edition, Crc Press, 2013.

[48] X.S. Miao, S.G. Chu, X.B. Xu, Degradation pathways of PCBs upon UV irradiation in hexane, Chemosphere 39 (1999) 1639-1650.

[49] M. Lores, M. Llompart, R. Gonzalez-Garcia, C. Gonzalez-Barreiro, R. Cela, Photolysis of polychlorinated biphenyls by solid-phase microextraction - "on-fibre" versus aqueous photodegradation, J. Chromatogr. A 963 (2002) 37-47.

[50] J. Boers, E. Leer, L. Gramberg, J. Koning, Levels of coplanar PCB in flue gases of high temperature processes and their occurrence in environmental samples, Fresenius J. Anal. Chem. 348 (1994) 163-166.

[51] C. Li, M.H. Zheng, L.R. Gao, B. Zhang, L.D. Liu, K. Xiao, Levels and distribution of $\mathrm{PCDD} / \mathrm{Fs}$, dl-PCBs, and organochlorine pesticides in sediments from the lower reaches of the Haihe River basin, China, Environ. Monit. Assess. 185 (2013) 1175-1187.

[52] D.C. Leggett, L.V. Parker, Modeling the equilibrium partitioning of organic contaminants between PTFE, PVC, and groundwater, Environ. Sci. Technol. 28 (1994) 1229-1233.

[53] S. Endo, A.A. Koelmans, Sorption of Hydrophobic Organic Compounds to Plastics in the Marine Environment: Equilibrium, Springer Berlin Heidelberg, Berlin, Heidelberg, 2016, pp. 1-20.

[54] F. Wang, C.S. Wong, D. Chen, X.W. Lu, F. Wang, E.Y. Zeng, Interaction of toxic chemicals with microplastics: a critical review, Water Res. 139 (2018) 208-219.

[55] G.W. Ehrenstein, Polymeric Materials: Structure, Properties, Applications, Carl Hanser Verlag GmbH Co KG, 2012.

[56] K. Duis, A. Coors, Microplastics in the aquatic and terrestrial environment: sources (with a specific focus on personal care products), fate and effects, Environ. Sci. Eur. 28 (2016) 2 .

[57] I.A. O'Connor, L. Golsteijn, A.J. Hendriks, Review of the partitioning of chemicals into different plastics: consequences for the risk assessment of marine plastic debris, Mar. Pollut. Bull. 113 (2016) 17-24.

[58] J.F. Li, H. Dong, D.H. Zhang, B. Han, C.J. Zhu, S.P. Liu, X.M. Liu, Q.Y. Ma, X.G. Li, Sources and ecological risk assessment of PAHs in surface sediments from Bohai Sea and northern part of the Yellow Sea, China, Mar. Pollut. Bull. 96 (2015) 485-490.

[59] Wikipedia, Polystyrene, in, 2018.

[60] B.G. Kwon, K. Saido, K. Koizumi, H. Sato, N. Ogawa, S.Y. Chung, T. Kusui, Y. Kodera, K. Kogure, Regional distribution of styrene analogues generated from polystyrene degradation along the coastlines of the North-East Pacific Ocean and Hawaii, Environ. Pollut. 188 (2014) 45-49. 
[61] Y. Yanagiba, Y. Ito, O. Yamanoshita, S.Y. Zhang, G. Watanabe, K. Taya, C.M. Li, Y. Inotsume, M. Kamijima, F.J. Gonzalez, T. Nakajima, Styrene trimer may increase thyroid hormone levels via down-regulation of the aryl hydrocarbon receptor (AhR) target gene UDP-glucuronosyltransferase, Environ. Health. Persp. 116 (2008) $740-745$.

[62] S. Hong, J. Lee, C. Lee, S.J. Yoon, S. Jeon, B.O. Kwon, J.H. Lee, J.P. Giesy, J.S. Khim, Are styrene oligomers in coastal sediments of an industrial area aryl hydrocarbon-receptor agonists? Environ. Pollut. 213 (2016) 913-921.
[63] K. Tanaka, H. Takada, R. Yamashita, K. Mizukawa, M. Fukuwaka, Y. Watanuki, Facilitated leaching of additive-derived PBDEs from plastic by seabirds' stomach oil and accumulation in tissues, Environ. Sci. Technol. 49 (2015) 11799-11807.

[64] A. Bakir, S.J. Rowland, R.C. Thompson, Enhanced desorption of persistent organic pollutants from microplastics under simulated physiological conditions, Environ. Pollut. 185 (2014) 16-23.

[65] Y.-T. Hung, L.K. Wang, H.H. Lo, C. Yapijakis, Hazardous Industrial Waste Treatment, CRC Press, 2006. 